\title{
HDL-PARTICLES SEPARATION EMPLOYING DIFFERENT PRECIPITATING AGENTS: FUNCTIONAL PROPERTIES OF THE IMPACT OF CHEMICAL PRECIPITATION ON LIPOPROTEIN PARTICLE-SIZE AND PARAOXONASE-1-ACTIVITY
}

\author{
Vanessa R. A. e Silva ${ }^{a}$, Elaine C. Albuquerque ${ }^{c}$, Ana Paula C. dos Santos ${ }^{\mathrm{a}, \mathrm{b}}$, Julio C. A. Santos ${ }^{\mathrm{a}}$, Lazaro S. S. Junior ${ }^{\mathrm{a}, \mathrm{b}}$, \\ William A. Presadaf, Carmen G. C. de M. Vinagre ${ }^{\mathrm{d}, \mathrm{e}}$ and Ricardo D. Couto ${ }^{\mathrm{a}, \mathrm{d}, *, \mathbb{D}}$ \\ aDepartamento de Análises Clínicas e Toxicológicas, Faculdade de Farmácia, Universidade Federal da Bahia, 40170-115 Salvador \\ - BA, Brasil \\ ${ }^{b}$ Hospital Naval de Salvador, Marinha do Brasil, 40010-000 Salvador - BA, Brasil \\ 'Departamento de Engenharia Química, Escola Politécnica, Universidade Federal da Bahia, 40210-630 Salvador - BA, Brasil \\ 'Laboratório de Metabolismo de Lípides, Instituto do Coração, Universidade de São Paulo, 05403-900 São Paulo - SP, Brasil \\ eUniversidade de Santo Amaro, 04743-030 São Paulo - SP, Brasil \\ fFundação Instituto de Administração, São Paulo, 01310-000 São Paulo - SP, Brasil
}

Recebido em 07/04/2019; aceito em 30/07/2019; publicado na web em 11/09/2019

\begin{abstract}
First-generation methods used to quantify HDL-cholesterol have been used for decades. Therefore, first-generation precipitatingreagents still in use, the chemical impact on HDL-particles functionality (structure/activity-relationship) is not well-documented. The study aim was to identify the most efficient precipitating-reagent for lipoprotein separation to be used in the HDL functionalevaluation. A cross-sectional design with convenience sampling was conducted. Plasma samples from 40 individuals, both genders, were evaluated. Biochemical analysis was performed after using different chemical precipitants (e.g. polyethylene glycol - PEG (P1), phosphotungstic-acid (P2), and dextran-sulfate/ $\mathrm{MgCl}_{2}(\mathrm{P} 3)$ ) to evaluate HDL-particle-size and PON1-activity. Parametric and non-parametric tests were performed using GraphPad-Prism 5.01 (USA). Differences were considered significant when a $\mathrm{p}$-value $<0.05$ were obtained. The PON1-activity comparison differed after $\mathrm{NaCl}$ stimulation ( $\mathrm{p}<0.001$, paired t-test) and among chemical precipitant treatments. The PON1-activity differed after sample precipitation with P1 when compared to P2, and P3 ( $\mathrm{p}<0.05$; ANOVA), before and after stratification for dyslipidemia group. The HDL particle-size comparison did not differ, but on the other hand, polydispersity differed after sample precipitation with P3 when compared to P1 or P2 ( $<<0.05$; ANOVA). DextranSulfate $/ \mathrm{MgCl}_{2}$ was a better chemical precipitant for PON1-activity, and PEG for HDL-particle-size, respectively. The HDL functional evaluation depends on the appropriately selected chemical precipitant.
\end{abstract}

Keywords: HDL-functionality; chemical precipitants; lipoprotein properties.

\section{INTRODUCTION}

Laboratory measurements of plasma lipoproteins and their lipid levels have an important role in research, clinical practices for lipid-lowering therapies and for both cardiovascular risk assessment and monitoring. Consequently, there has been a continuos effort to develop and improve methods that can be used to consistently measure plasma lipoproteins and their lipid content. ${ }^{1}$ High-density lipoprotein cholesterol (HDL-c) has been used as a clinical parameter and is commonly requested by physicians to assess the risk correlated with cardiovascular diseases. $^{2}$

To determine HDL-c an analytical system capable to detect only the cholesterol transported into the high-density lipoproteins (HDL) must be used. Methods for HDL-c determination explore the physical and chemical characteristics inherent to apoA and apoB-containing lipoproteins as well as the artifacts used to inhibit the participation of low-density (LDL), very-low-density (VLDL) lipoproteins and chylomicrons in plasma cholesterol determination reactions. The different methods include separation by ultracentrifugation, apolipoprotein (apo) B-lipoprotein precipitation, apoB-lipoproteins complexation by anti-apoB antibodies, modification of enzyme affinity to decrease interaction with apoB-lipoproteins, among others. ${ }^{3}$

*e-mail: rdc@ufba.br
The first method used to HDL-c determinations was cumbersome, with multiple steps and requiring several ultracentrifugation steps. Separation of lipoproteins by ultracentrifugation for further quantifications is a laborious and time-consuming method and requires the use of special centrifuges with high rotation speeds, as well as the use of salts for final density adjustments, a step that can cause lipoprotein structural alterations. While ultracentrifugation is very useful in research - the gold standard technique for HDL separation from other lipoproteins, and its sub-populations - it is not considered a practical methodology for routine laboratory analysis. New methods were introduced, and they can be divided into three generations: the 1st generation - precipitation methods; the 2nd generation - automated precipitation methods; and the 3rd generation - homogeneous (direct) assays. ${ }^{1}$

First generation methods or chemical precipitation used polyanions, sometimes combined with bivalent cations, or nonionic polymers, which form insoluble aggregates with low-density lipoproteins, leaving the HDL-particle in solution. This particle can then be recovered by decantation or undernatant pipetting, prior to HDL-c measurement. Insoluble proteins can be precipitated by lowspeed centrifugation. ${ }^{4}$

Second generation methods were created with the purpose of substituting the centrifugation process. The methodologies used combined polyanions and bivalent cations with magnetic particles, in order to facilitate apoB-rich lipoproteins removal, alongside the development of systems that could be used with a previously 
calculated amount of precipitating agents, ready to be inserted into automatic analyzers, which used reactive tapes impregnated with precipitating agents. ${ }^{1}$

Homogenous methods or third generation methods used different techniques for apoB-lipoproteins segregation. These methods were based on anti-apoB antibodies that formed complexes with chylomicrons, VLDL, LDL and blocked the enzymes action, such as cholesterol esterase and cholesterol oxidase or were based on the use of polyanions, which aggregated apoB-lipoproteins. A surfactant acted only on HDL-particles, exposing its content to the action of cholesterol esterase and cholesterol oxidase. The synthetic polyanion aggregation action was evaluated by transmission electron microscopy, which proved to be effective only against apoB-lipoproteins. ${ }^{1}$

HDL has antiatherogenic properties which includes antioxidant and antiinflamatory actions. Efflux of cholesterol from the cells to HDL is associated with the presence of coronary artery disease. ${ }^{2}$ In addition to the role in reverse cholesterol transport, HDL has several other antiatherogenic functions, such as antioxidant, and antiinflammatory functions, ${ }^{5,6}$ that can be, at least in part, independent of HDL-c concentrations..$^{2,5-7}$ Thus, functional and metabolic tests are needed to fully evaluate HDL anti-atherosclerotic protection and for this, one needs a practical and confiable method to obtain HDL from plasma.

Since some reagents used to precipitate apoB-containing lipoproteins may interfere with HDL particle-size, and particle functionality, ${ }^{8}$ regarding the importance of HDL particles-remodeling and antioxidant-activity, this study aimed to identify the most efficient chemical precipitating reagent to be used for lipoproteins separation to perform HDL functional evaluations.

\section{EXPERIMENTAL}

\section{Study design and sampling}

An observational, cross-sectional study, with convenience sampling, was carried out to obtain serum and plasma samples from healthy individuals admitted for laboratory exams at the Clinical Chemistry Laboratory from the Faculty of Pharmacy at the Federal University of Bahia. To identify the significant number of samples for the study, a sample size calculation was conducted (WINPEPI for Windows software; version 11.48). The study included samples from 40 individuals of both genders, between 40 and 70 years old. The individuals were separated into 4 groups, according to their lipid profile, as: HypTRI: Isolated hypertriglyceridemia ( $\mathrm{TG}>150 \mathrm{mg} \mathrm{dL}^{-1}$ ); HypLIP: Mixed hyperlipidaemia (LDL-c $>160 \mathrm{mg} \mathrm{dL}^{-1}$ and $\mathrm{TG}>150 \mathrm{mg} \mathrm{dL}^{-1}$ ); Low HDL-c (men $<40 \mathrm{mg} \mathrm{dL}^{-1}$ and women $<50 \mathrm{mg} \mathrm{dL}^{-1}$ ); Normo: normolipidemidemic ( $\mathrm{TG}<150 \mathrm{mg} \mathrm{dL}^{-1}$, LDL-c $<130 \mathrm{mg} \mathrm{dL}^{-1}$ and HDL-c $>40 \mathrm{mg} \mathrm{dL}^{-1}$ (men) and $>50 \mathrm{mg} \mathrm{dL}^{-1}$ (women).

The study was approved by the Climério de Oliveira Maternity Hospital Research Ethics Committee, UFBA (CEP/MCO/UFBA), document N.379 (27/09/2005) as part of an Additive Resolution N.029/2014-2016.

*Inclusion criteria: Blood samples donated by individuals of both genders, between 40 and 70 years-old, with or without dyslipidemias. All participants signed a Free Prior Informed Consent (FPIC) form;

*Non-inclusion criteria: Blood samples donated by individuals using lipid-lowering drugs, and/or that refused to sign the FPIC form.

\section{Laboratory determinations}

\section{Biochemical Determinations}

Blood samples were collected after a $12 \mathrm{~h}$ overnight fast for serum determinations of several biochemical laboratory parameters. Total cholesterol (TC) concentrations, and triglycerides (TG) were determined by enzymatic colorimetric methods. HDL-c was measured using the same method used for total cholesterol after lipoprotein precipitation with magnesium-phosphotungstate. The VLDL-c and LDL-c concentrations were calculated by Friedewald, ${ }^{9}$ for TG values until $399 \mathrm{mg} \mathrm{dL}^{-1}$. All determinations were performed on the automated LABMAX 560 equipment (Labtest diagnostic S/A, Brazil). The selected samples were stratified according to the V Brazilian Guideline Actualization on Dyslipidemias and Atherosclerosis Prevention ${ }^{10}$ in Normolipidemic and Dyslipidemic (isolated hypercholesterolemia, isolated hypertriglyceridemia, mixed hyperlipidemia and low HDL-c).

\section{Lipoprotein precipitating solutions}

HDL was separated from apoB-containing lipoproteins by chemical precipitation. For HDL isolation and subsequent experiments, each sample was precipitated with three different precipitating solutions, those being, polyethylene glycol (PEG) $8000\left(200 \mathrm{~g} \mathrm{~L}^{-1}\right)$ in $0.2 \mathrm{~mol} \mathrm{~L}^{-1}$ glycine buffer adjusted to $\mathrm{pH} 10$ with $\mathrm{NaOH}(\mathrm{P} 1)$; phosphotungstic acid-Ca ${ }^{2+}\left(3 \mathrm{~g} \mathrm{~L}^{-1}\right)(\mathrm{P} 2)$; and dextran sulfate/ $\mathrm{MgCl}_{2}\left(15 \mathrm{~g} \mathrm{~L}^{-1}\right)(\mathrm{P} 3)$, by mixing $500 \mu \mathrm{L}$ of each precipitating solution to $500 \mu \mathrm{L}$ of EDTA plasma and stirred in a vortex-mixer for $30 \mathrm{~s}$. Samples were then centrifuged at $4{ }^{\circ} \mathrm{C}$ for $10 \mathrm{~min}$ at $1250 \mathrm{~g}$ in an Eppendorf® microcentrifuge to obtain the supernatant.

\section{Paraoxonase (PON1) activity}

The PON1-activity was spectrophotometrically measured with a 96-well SIRIO Microplate Reader Seac. SrL (Firenze, IT), using a method described by Macknsess et al. ${ }^{11}$ and Senti et al. ${ }^{12}$ The reaction is based on paraoxon hydrolysis with p-nitrophenol and diethylphosphate formation. To measure PON1 basal activity, in each plate well, $7 \mu \mathrm{L}$ of serum samples were added to a solution of $140 \mu \mathrm{L}$ of Tris- $\mathrm{HCl} 0.1 \mathrm{mmol} \mathrm{L}^{-1}, \mathrm{CaCl}_{2} 2 \mathrm{mmol} \mathrm{L}^{-1}$, and paraoxon $1.129 \mathrm{mmol} \mathrm{L}^{-1}$ (dietil-4-nitrofenil phosphate; Sigma Chemical, St. Louis, MO, USA). To measure $\mathrm{NaCl}$ stimulated PON1 activity, $7 \mu \mathrm{L}$ of each obtained supernatant, after the use of precipitating reagents, was pipetted into the same solution used to measure basal activity plus adding $\mathrm{NaCl} 1 \mathrm{~mol} \mathrm{~L}^{-1}$. Six readings were taken at one-minute intervals in order to calculate PON1 basal and stimulated activities. The results were achieved by multiplying the mean absorbance variation by a calculated factor: factor $=$ total reaction volume (TRV) (mL) / 8405 x sample value (SV) $(\mathrm{mL}) \mathrm{x}$ cuvette thickness (cm), in which $\varepsilon 405^{-1805} \mathrm{~mL}^{-1} \mathrm{~cm}^{-1}$. Therefore, PON1 activity (basal or stimulated) $=$ factor $\times \Delta \mathrm{abs} /$ minute.

\section{HDL particle-size}

The HDL particle-size was measured by laser light scattering (LLS), using a ZetaPALS Zeta Potential Analyzer (ZetaPALS; Brookhaven Instruments, Holtsville, NY) accordingly to the method described by Lima and Maranhão ${ }^{8}$ at the Supercritical Nanotechnology Laboratory (LNS) from Polytechnic School, UFBA. $10 \mathrm{~mL}$ of blood were collected from all subjects by a vacuum dispositive into a glass tube containing EDTA (k3E k3-EDTA, VACUETTE $^{\circledR}$ ). Plasma was then obtained by centrifugation at $4{ }^{\circ} \mathrm{C}$ for $15 \mathrm{~min}$ at $1250 \mathrm{~g}$. For the isolation of HDL and subsequent sizing by LLS, apoB containing lipoproteins from each sample was precipitated by three different precipitating solutions as mentioned above. A $0.5 \mathrm{~mL}$ portion of each supernatant was added to $1.5 \mathrm{~mL}$ 
Table 1. Lipid profile descriptive data from different groups, with and without dyslipidemia

\begin{tabular}{|c|c|c|c|c|c|}
\hline & $\mathrm{TC}\left(\mathrm{mg} \mathrm{dL}^{-1}\right)$ & HDL-c $\left(\mathrm{mg} \mathrm{dL}^{-1}\right)$ & LDL-c $\left(\mathrm{mg} \mathrm{dL}^{-1}\right)$ & VLDL-c $\left(\mathrm{mg} \mathrm{dL}^{-1}\right)$ & $\mathrm{TG}\left(\mathrm{mg} \mathrm{dL}^{-1}\right)$ \\
\hline Normo $(n=21)$ & $199 \pm 27$ & $70 \pm 17$ & $108 \pm 26$ & $21 \pm 5$ & $102 \pm 24$ \\
\hline HypTRI (n = 8) & $212 \pm 29$ & $61 \pm 11$ & $110 \pm 31$ & $38 \pm 15$ & $209 \pm 40$ \\
\hline Hyp LIP (n = 2) & $289 \pm 13$ & $64 \pm 8$ & $185 \pm 12$ & $40 \pm 9$ & $198 \pm 46$ \\
\hline Low HDL-c $(\mathrm{n}=9)$ & $183 \pm 35$ & $40 \pm 8$ & $104 \pm 30$ & $39 \pm 15$ & $193 \pm 76$ \\
\hline
\end{tabular}

of $\mathrm{NaCl}\left(10 \mathrm{mmol} \mathrm{L}^{-1}\right)$, passed through a $0.22 \mu \mathrm{m}$ filter (Millipore Products Division) so to exclude any undesirable particles, and then dispensed into a disposable cuvette. The ZetaPALS Zeta Potential Analyzer used a $29 \mathrm{~mW}$ helium-neon laser at $658 \mathrm{~nm}$ to excite the samples. Scattered light was collected at a $90^{\circ}$ angle by a photoncounting photomultiplier tube, and then directed to a correlator. The BIC particle sizing software derives particle sizes from the correlator function. Results of each sample were expressed as the mean, which is the harmonic intensity-averaged particle diameter. All experiments were performed at $25^{\circ} \mathrm{C}$, and the results are expressed by the means of five runs of 2 min each.

\section{Statistical analysis}

Data were analyzed with descriptive and inferential statistics. To test data distribution, a D' Agostino normality test was employed. Paired variance analysis (dependent samples) was performed according to the type of data distribution, followed by multiple comparison post hoc test. All tested data were considered significant when the critical $(\mathrm{p})$ value was under 0.05 to a confidence interval of 0.95 . The statistical analyses were done with a GraphPad Prism software, version 5.01 (USA) statistical package.

\section{RESULTS}

Table 1 shows the descriptive data of TC, HDL-c, VLDL-c, LDL-c and TG lipid profile samples serum concentrations stratified by normolipidemic and dyslipidemia groups according to the $\mathrm{V}$ Brazilian Guideline on Dyslipidemias and Atherosclerosis. ${ }^{10}$

The PON1-activity comparison, basal and $\mathrm{NaCl}$ stimulated assays, is shown in the figure 1 . The PON1-activity was greater in the $\mathrm{NaCl}$ stimulated when compared to basal assay $(\mathrm{n}=21 ; \mathrm{p}<0.001)$. All assays were done in triplicate.

Figure 2 shows the comparison among PON1-activities stimulated by $\mathrm{NaCl}$ after apoB-rich lipoprotein precipitating solutions. The

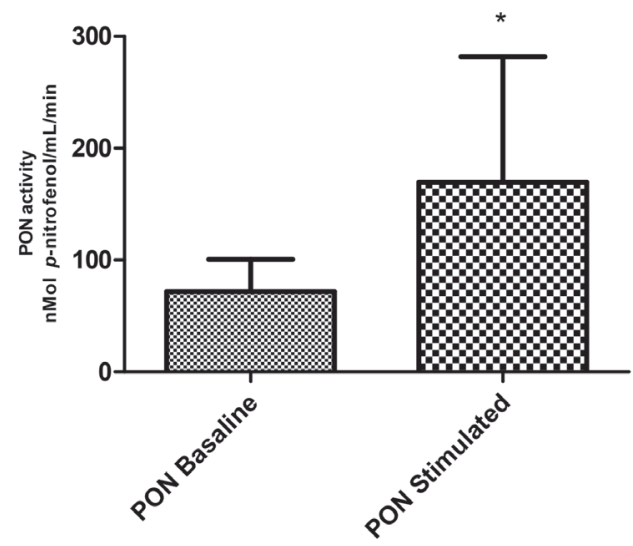

Figure 1. PONI activity, basal and NaCl stimulated. There was a significant difference $(n=21$; paired $t$-test; $p<0.001)$. Assays were performed in triplicate. PON1 baseline: Basal Paraoxonase activity; PON1 stimulated: Stimulated Paraoxonase activity with $\mathrm{MgCl}_{2}$ result obtained by $\mathrm{P} 2$ was greater than $\mathrm{P} 1 ; \mathrm{P} 3$ was also greater when compared to P1 ( $\mathrm{n}=21$; one-way ANOVA, paired test, Tukey post hoc test, $\mathrm{p}<0.001)$. All assays were done in triplicate.

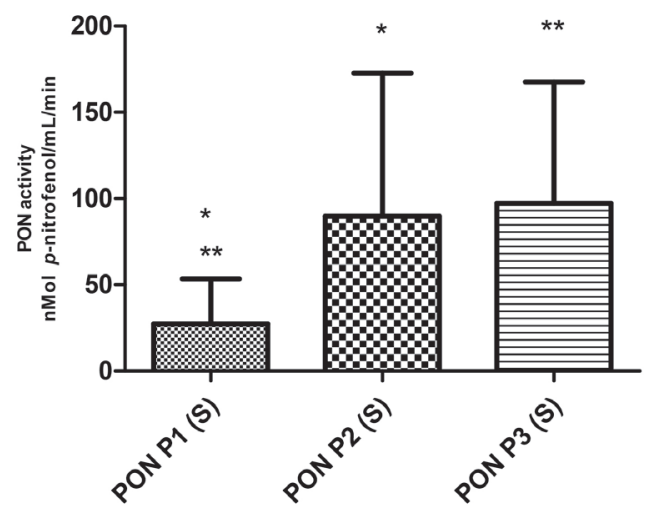

Figure 2. PON1 NaCl stimulated (S) activity, after precipitation of apoB-rich lipoproteins using different precipitating solutions. There was a significant difference ( $n=21$; one-way, ANOVA, paired test, Tukey post hoc test, $p<0.001$ ) between $P O N 1$ activity obtained after the use of $P 1$ and $P 2$ precipitants, as well as between the $P O N 1$ activity obtained after the use of $P 1$ and $P 3$ precipitants. The difference was not significant between PON P2 and P3. Assays were performed in triplicate. $P O N \mathrm{P} 1$ : paraoxonase activity stimulated by $\mathrm{NaCl}$ after using polyethylene glycol; PON P2: paraoxonase activity stimulated by NaCl using Phosphotungstic acid; PON P3: paraoxonase activity stimulated by $\mathrm{NaCl}$ using Dextran Sulfate/ $\mathrm{MgCl}_{2}$

The PON1 NaCl stimulated activities after apoB-rich lipoproteins precipitation by different solutions (P1, P2, and P3) from none and dyslipidemia different groups are shown in Figure 3. In the Isolated Hypertrigliceridemia group, the result obtained by P3 was greater than P1. In the Normolipidemic and in the Low HDL-c groups, P2 and P3 were greater than P1 (one-way, ANOVA, paired test, Tukey post-test, $\mathrm{p}<0.001$ ). The Mixed Hyperlipidemia group data did not participate on comparison, $\mathrm{n}=2$. All assays were done in triplicate.

Figure 4 shows the HDL particle-size determined after precipitation by different solutions in none and dyslipidemia different groups. There was no statistically significant difference among the groups $(\mathrm{p}>0.05)$. The data from the mixed hyperlipidemia group did not participate on comparison. All assays were done in triplicate.

Figure 5 shows the polydispersity (PDI) measure in different groups of dyslipidemias. In the normolipidemic group, the PDI obtained by P3 was greater than P1 and P2. In the Low HDL-c group, P2 and P3 were greater than P1. In the Hypertrigliceridemic and in the Mixed Hyperlipidemia groups, there was no difference in solution polydispersity (one-way, ANOVA, paired test, Tukey posttest, $\mathrm{p}<0.001)$. All assays were done in triplicate.

\section{DISCUSSION}

Changes in plasma lipids and their lipoproteins are associated with elevated cardiovascular risk. Cholesterol elevations associated with 

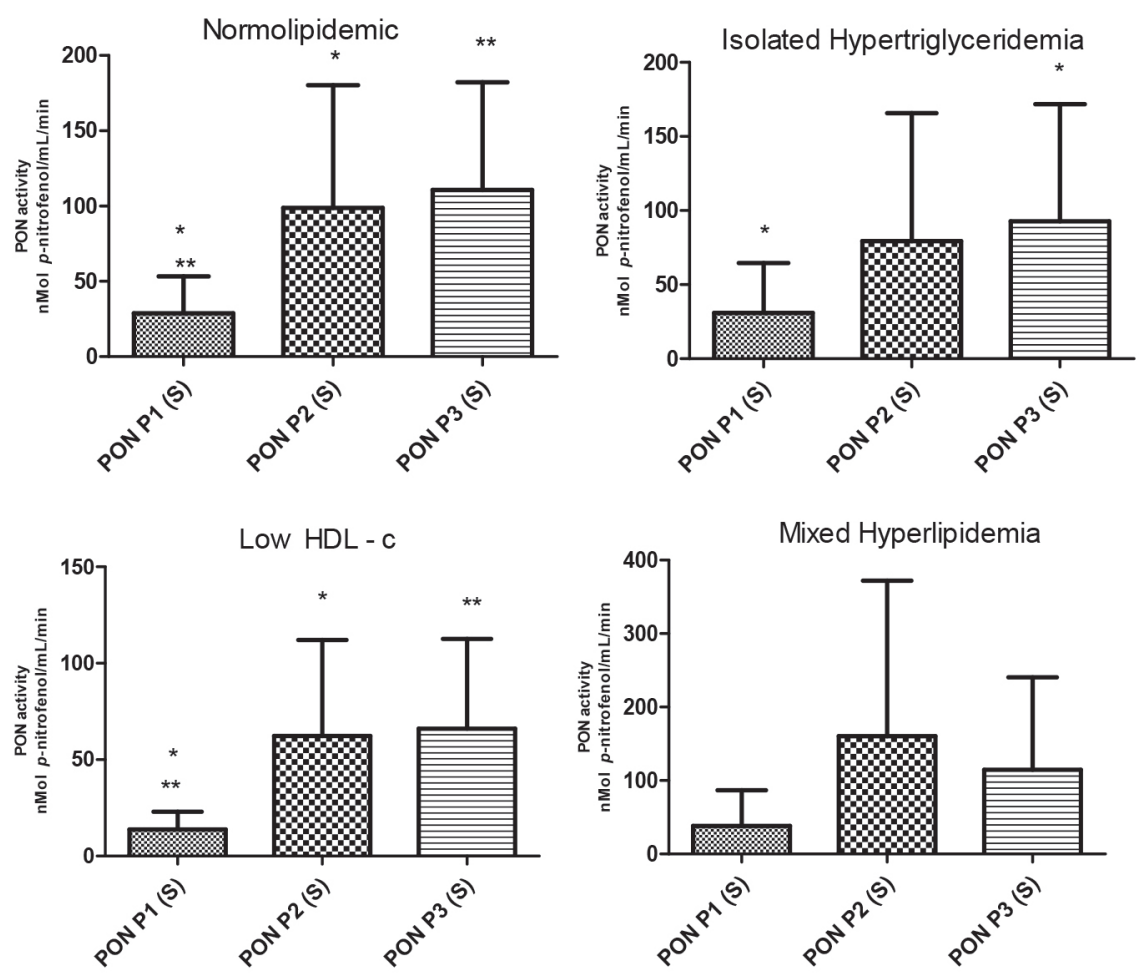

Figure 3. PON1-activity stimulated by NaCl, using different precipitating solutions in different groups of dyslipidemias. (A) There was a significant difference ( $n=21$; one-way, ANOVA, paired test, Tukey post-test, $p<0.001$ ), only between the PON P1 and P3 groups. The difference was not significant between PON $P 2$ and $P 3$ groups, and PON P1 and P2 groups. (B) There was a significant difference $(n=8$; one-way, ANOVA, paired test, Tukey post-test, $p<0.001)$ between $P O N P 1$ and $P 2$ groups and $P O N P 1$, and $P 3$ groups. There was no significant difference between PON $P 2$ and $P 3$ groups. (C) There was a significant difference ( $n=9$; one-way, ANOVA, paired test, Tukey post-test, $p<0.001)$ between PON P1 and P2 groups, and PON P1 and P3 groups. The difference was not significant between PON P2 and P3 groups. (D) Only descriptive data, statistical analysis were not done, $n=2$. Assays were performed in triplicate. PON P1: paraoxonase activity stimulated by $\mathrm{NaCl}$ using a polyethylene glycol; PON P2: paraoxonase activity stimulated by NaCl using Phosphotungstic acid; PON P3: paraoxonase activity stimulated by $\mathrm{NaCl}$ using Dextran Sulfate/ $\mathrm{MgCl}_{2}$
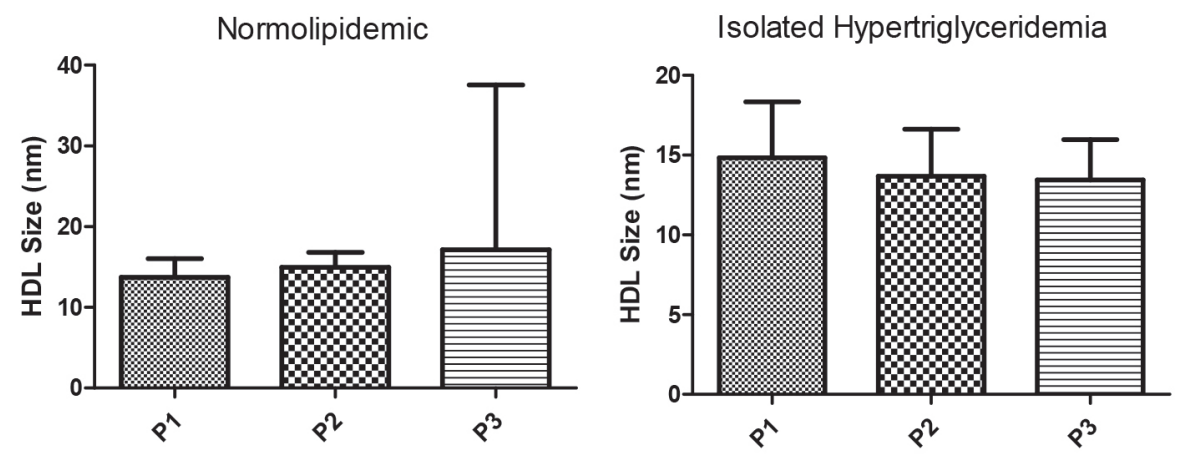

$$
\text { Low HDL - c }
$$
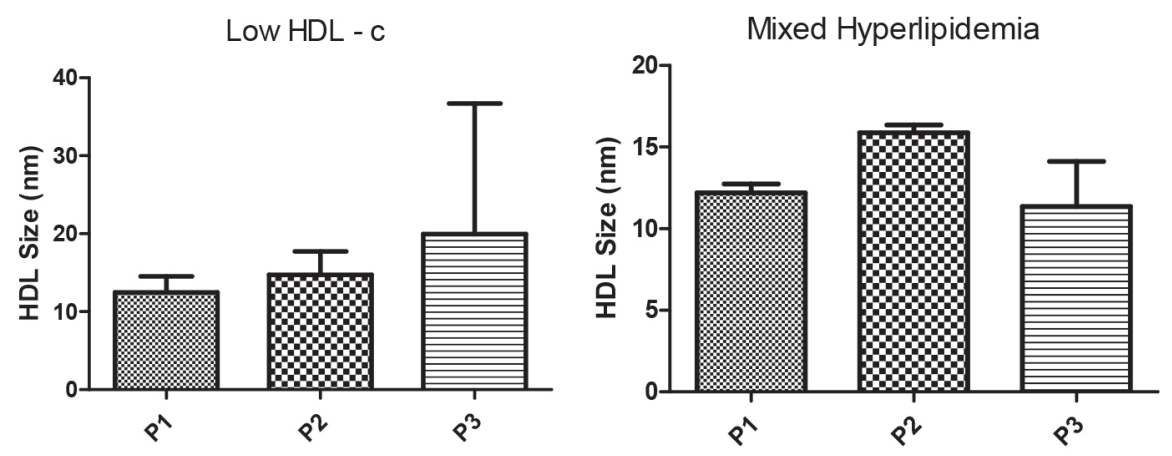

Figure 4. HDL particle size determined after precipitation by different solutions in different groups of dyslipidemias. No significant difference among the groups was found (one-way, ANOVA, paired test, Tukey post hoc test, $p=0.46$ ). The mixed hyperlipidemia condition were not compared among groups, $n=2$. P1: HDL particle size using polyethylene glycol; P2: HDL particle size using phosphotungstic acid; P3: HDL particle size using Dextran Sulfate/MgCl. Assays were performed in triplicate. (A) Normolipidemic $(n=21)$; (B) Isolated Hypertriglyceridemia $(n=8)$; (C) Low HDL-c $(n=9)$; $(D)$ Mixed Hyperlipidemia $(n=2)$ 
Normolipidemic

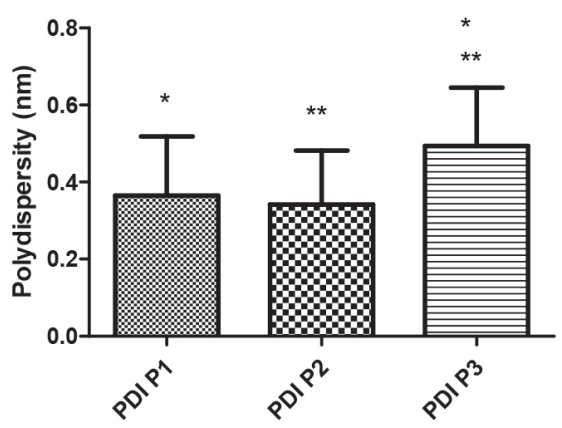

Mixed Hyperlipidemia

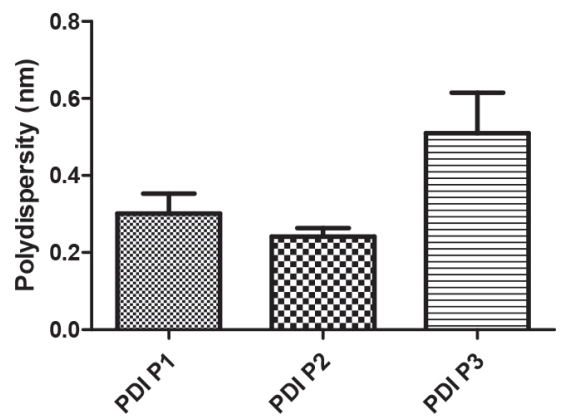

Isolated Hypertriglyceridemia

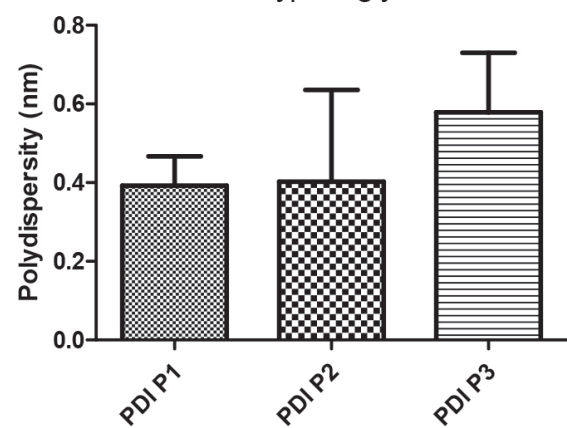

Low HDL - c

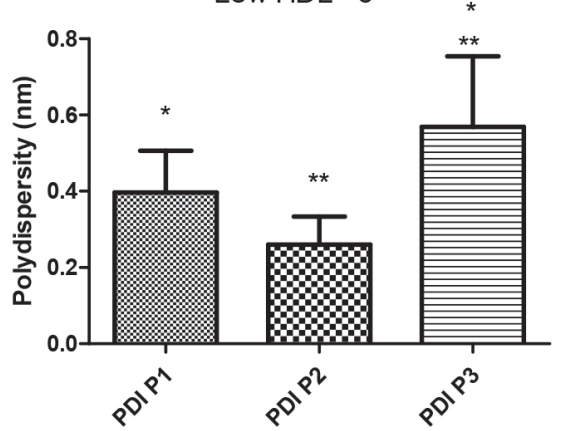

Figure 5. Polydispersity of the solution used to determine HDL particle size after precipitation by different solutions in different groups of dyslipidemias. (A) There was a significant difference ( $n=21$; one-way, ANOVA, paired test, Tukey post-test, $p<0.001)$ between PDI P1 and P3 groups and PDI P2 and P3 groups. The difference was not significant between P1 and P2 PDI groups. (B) No significant difference between groups was found ( $n=8)$. (C) There was a significant difference ( $n=9$; one-way, ANOVA, paired test, Tukey test, $p<0.001)$ between P1 and P3 PDI groups and P2 and P3 PDI groups. The difference was not significant between $P 1$ and P2 PDI groups. (D) Only descriptive data, the mixed hyperlipidemia condition were not compared among groups, $n=2$. Assays were performed in triplicate. PDI P1: Polydispersity of the solution using Polyethyleneglycol; PDI P2: Polydispersity of the solution using Phosphotungstic acid; PDI P3: Polydispersity of the solution using Dextran Sulfate/ $\mathrm{MgCl}_{2}$. (A) Normolipidemic; (B) Isolated Hypertriglyceridemia; (C) Low HDL-c; (D) Mixed Hyperlipidemia

low-density lipoprotein (LDL) are closely correlated with increased cardiovascular risk. ${ }^{13}$ According to the V Brazilian Guideline actualization on Dyslipidemia and Atherosclerosis Prevention, ${ }^{10}$ lipoproteins constitute a heterogeneous class of particles. There is evidence suggesting that the different subclasses of LDL and HDL can increase early on atherosclerosis development, due to changes in its size, composition, and functionality. Alterations in lipoproteins structure observed in the presence of dyslipidemias may also modify the action of chemical precipitants commonly used for the separation of these particles containing apoA and apoB in the routine laboratory.

One of the main ways to isolate LDL and VLDL lipoproteins from serum is the combination of glycosaminoglycan's and bivalent cations. This association leads to insoluble complexes formation that can be isolated by conventional centrifugation. The separation process showed adequate specificity without promoting alteration in lipoprotein structure, and as such, ultracentrifugation was not required. ${ }^{14}$ Srinvasan et al. ${ }^{15}$ studied the selectivity of the interaction among HDL, LDL and VLDL lipoproteins in the presence of heparin and $\mathrm{Ca}^{++}, \mathrm{Mg}^{++}$and $\mathrm{Mn}^{++}$. The study demonstrated that $\mathrm{Ca}^{++}$and $\mathrm{Mg}^{++}$ cations lead to insoluble complexes formation with LDL and VLDL, different from $\mathrm{Mn}^{++}$cation that promotes the HDL, LDL and VLDL precipitation in the presence of heparin. All of those processes that drives to lipoprotein insoluble complexes formation are chemically made, on the other hand, HDL lipoprotein can be separated by using only its physical or physical-chemical properties (e.g. density, size, and charge), which could be normally achieved by equipment utilization such as ultracentrifuges, electrophoresis platforms, fast liquid chromatography, nuclear magnetic resonance, and it's technic variations. $^{4}$
In this study the HDL particle antioxidant activity was evaluated by PON1 activity determination. The PON1 activity had a significant increase after $\mathrm{NaCl}$ addition. A study by Eckerson et al. ${ }^{16}$ showed that the PON1 activity in humans is stimulated by high $\mathrm{NaCl}$ concentrations. The physiological significance of PON1 activation by $\mathrm{NaCl}$ is remains unclear. Some studies have suggested that PON1 activities, basal and $\mathrm{NaCl}$ stimulation, are differentially affected under pathological conditions. Schiavon et al. ${ }^{17}$ found that basal enzyme activity, unlike that stimulated by $\mathrm{NaCl}$, is reduced in hemodialyzed patients. These data suggest that, under certain conditions, basal PON1 activity is more sensitive to modifying factors than stimulated activity by $\mathrm{NaCl} .{ }^{18}$

The PON1 activity after treatment with different precipitant solutions, samples with or without stratification by dyslipidemia group, behaved differently. Several factors can alter PON1 activity such as environmental and inherent polymorphisms that can affect enzyme activity. The exogenous interfering factors, lipemic or hemolyzed samples can also be linked to high variability in PON1 activity among individuals. ${ }^{3}$

The study conducted by Costa et al. ${ }^{3}$ concluded that the phosphotungstic polyanion in addition to dextran sulfate selectively inhibit the action of enzymes in homogeneous systems for HDL-c determinations. Costa et al. ${ }^{3}$ also verified that chemical modifications of the enzyme action changes the interaction between HDL and LDL. Therefore, comparing our results with those obtained by Costa et al. ${ }^{3}$ for enzyme activity in HDL metabolism, this activity behaved similarly when exposed to the same precipitants used in our study.

High-density lipoprotein is composed of heterogeneous particles ranging in size from 7 to $14 \mathrm{~nm}$. There is evidence that suggests that 
most of the HDL cardioprotective properties are associated with the $\mathrm{HDL}_{2}$ fraction (larger particles) rather than the $\mathrm{HDL}_{3}$ fraction (smaller particles) in patients with coronary artery disease. Various methods, such as ultracentrifugation, chemical precipitation, immunoaffinity chromatography, electrophoresis, among others, have been used to separate HDL subfractions. HDL particle size determination by laser light scattering (LLS) can be performed after apoB-containing lipoproteins chemical precipitation. This approach has a practical advantage over ultracentrifugation or electrophoresis. ${ }^{8}$

The present study determined the HDL particle-size by laser light scattering (LLS), using various combinations of polyanions and bivalent cations to precipitate apoB-containing lipoproteins. PEG is a neutral polymer that reduces LDL and VLDL solubility, whereas the combination of polyvalent polyanions, such as heparin and dextran sulfate, interact with arginine and other positively charged residues on apolipoproteins. Cations such as manganese or magnesium help the polyanion-lipoprotein complexes to be formed from the interaction with phospholipids, resulting in precipitation. Dextran sulfate-magnesium chloride $\left(\mathrm{MgCl}_{2}\right)$ is the most common method used for quantifying HDL-c in most clinical laboratories. ${ }^{19}$

In this study no significant difference in particle size was observed in analyzed samples, even with the use of different precipitants. Similar behavior was evidenced after stratification by the dyslipidemia group. The use of PEG for apoB-containing lipoprotein precipitation, when compared to other precipitants tested, better approximated the results obtained by ultracentrifugation in HDL particle size. ${ }^{8}$ The use of PEG showed particle size results compatible with that expected for HDL, as well as the use of dextran sulfate and phosphotungistat- $\mathrm{Ca}^{++}$ based methods. Although dextran sulfate produced the largest HDL diameter polydispersity results when segregated in normolipidemic and low HDL-c groups, it was still within the normally accepted limits. The diameter polydispersity is a sample parameter for heterogeneity and quality.

The lipid characteristics inherent to each dyslipidemia profile can directly influence in the HDL particle size and functionality. These results of this study suggest that the use of precipitating solutions with different chemical characteristics may interfere with the lipoproteins structure and HDL particle-size measurements by LLS. According to Dias et al. ${ }^{20}$ precipitant concentrations may influence the apoBrich lipoproteins precipitation and consequently, in particle-size determination, can attribute larger diameter values to HDL particle aggregates.

In particle size, dispersion analyses are very important in order to distinguish the weak aggregates or agglomerates from individual particles. According to data established by Malvern, ${ }^{21}$ polydispersity index values lower than 0.1 indicate systems considered monodisperse while values greater than 0.7 indicate preparations with heterogeneous, therefore polydispersed, particles.

The data of this study shows that none of the analyzed groups had a polydispersity index higher than 0.7 . Therefore, none of the evaluated solutions showed characteristics of polydispersed solutions, although there was a significant difference between solutions precipitated with PEG and dextran sulfate, as well as between solutions precipitated with phosphotungstic acid and by dextran sulfate. The polydispersity showed no significant difference between the precipitating solutions, polyethylene glycol and phosphotungstic acid. When comparing the results after stratification by dyslipidemias, given the possibility of these profiles modifying the lipoproteins structures, neither the group characterized by isolated hypertriglyceridemia nor the group characterized by mixed hyperlipidemia showed significant polydispersity differences with the different precipitant solutions. On the other hand, the normolipidemic and low HDL-c groups showed significant differences depending on which precipitant were used.
The precipitants analyzed, in terms of impacts on HDL particle, structure and activity, behaved differently. There was no significant difference between the precipitants with respect to particle-size, but there was a significant difference in the polydispersity data analysis. In this case, the highest index being the samples treated with phosphotungstic acid. In parallel, the data that evaluated particle functionality, from its antioxidant characteristics, suggest a greater preservation of this characteristic with the use of precipitants such as dextran sulfate and phosphotungstic acid.

To differentiate the mechanisms of interactions between dextran sulfate with HDL, and with LDL, Kim and Nisada ${ }^{22}$ provide information about the charge characteristics of these lipoproteins. As stated by Srinvasan et al. ${ }^{15} \mathrm{Ca}^{++}$and $\mathrm{Mg}^{++}$cations lead to insoluble complexes formation with LDL and VLDL in the presence of heparin, but not with HDL particles. Although it appeared that primarily, the phospholipid components of HDL are involved in the formation of the insoluble complexes, the protein components, both apolipoproteins A-I (apoA-I) and A-II (apoA-II), reduced the HDL complex-forming ability as a result of its large number of free negative-charge, which differed from the LDL apoB protein positively-charged groups that even in the absence of $\mathrm{Ca}^{++}$cations lead to the formation of LDL and VLDL dextran sulfate insoluble complexes. Although there is an electrostatic balance between lipoprotein phospholipids and protein charges that interact with more sulfate groups of the dextran-sulfate, the increased presence of $\mathrm{Ca}^{++}$, strength the LDL phospholipid zwitterionic polar heads participation in the LDL-Dextran sulfate insoluble complex formation. Based on the above mechanism proposed, we can state that the absence of $\mathrm{Ca}^{++}$in the preparation, i.e. Dextran sulfate magnesium chloride $\left(\mathrm{MgCl}_{2}\right)$, supported by the review of Hafiane and Genest, ${ }^{4}$ from the five elected methods for HDL cholesterol quantification none of then use $\mathrm{Ca}^{++}$. As HDL-particle enzymes functionality depends on HDL solubility and dextran sulfate $\mathrm{MgCl}_{2}$ do not interact efficiently to it (has a disproportionate impact on large apoB-containing lipoproteins when compared to smaller HDL-particles), the absence of $\mathrm{Ca}^{++}$would be one of the chemical aspects to support the dextran-sulfate $\mathrm{MgCl}_{2}$ as a better precipitating reagent to be used for the evaluation of HDL antioxidant functional properties.

The data from the PEG reagent finds in our study give us the support to elect it to be used in the HDL particle-size, diameter measurement, when compared to dextran sulfate $\mathrm{MgCl}_{2}$, and phosphotungstic acid $\left(\mathrm{Ca}^{++}\right)$. Even though Davidson et al. ${ }^{19}$ by comparing the use of PEG for HDL particle-size measurements wouldn't achieve the same results. Firstly, they inform difficulties to measure HDL diameter by LLS and nuclear magnetic resonance (NMR) particle-sizing methodologies, telling that the PEG remains $80 \%$ in the solution after VLDL and LDL precipitation; secondly, they used asymmetric flow field-flow fractionation (AF4) instead of LLS, justifying it because AF4 particle-sizing methodology does not require a separation media like other technics. However, they observed a peak that represents HDL-sized particles and another one that possibly represents the presence of apoB particles upon precipitation with PEG. On the other hand, they affirm that with the use of dextran sulfate/ $\mathrm{MgCl}_{2}$ and heparin sodium/ $\mathrm{MnCl}_{2}$ the VLDL/ LDL peak disappeared, consistent with apoB precipitation. As we used LLS and they used AF4 methodology, the studies used different PEG concentrations, and they do not use phosphotungstic acid, only compared PEG with $\mathrm{MgCl}_{2}$ derived precipitants, our studies could not be completely compared.

The study of Davidson et al. ${ }^{19}$ bright to us very sharp information about the use of lipoprotein chemical precipitation and particle-sizing peculiar's methodology conditions. Thus, as we did not find other similar studies to compare our data, studies that bring comparable 
methodologies and conditions, we will address these studies differences to our future investigations.

\section{CONCLUSIONS}

The present study evaluated the influence of methodologies used for lipoprotein separation to measure HDL PON1 antioxidant activity and particle-size, functional analyses. There was an increase in the PON1-activity after $\mathrm{NaCl}$ stimulation, which is useful in particle functionality evaluation. When HDL particle functionality was evaluated by its antioxidant characteristic, the chemical precipitant that most preserved this characteristic was dextran sulfate. The particle structural evaluation by particle-size measurement, suggests that the precipitant that most preserved HDL particle structural characteristics was PEG. Therefore, it is important to carefully choose the most efficient apoB-rich lipoprotein precipitating reagent for the HDL functionality evaluation. That methodological concept would be also used in the diagnosis and subsidize the elucidation of clinical conditions associated to ineffective HDL particle reverse cholesterol transport and it's other functional protective pleiotropic properties.

\section{REFERENCES}

1. Warnick, G. R.; Nauck, M.; Rifai, N.; Clin. Chem. 2001, 47, 579.

2. Rohatgi, A.; Khera, A.; Berry, J. D.; Givens, E. G.; Ayers, C. R.; Wedin, K. E.; Neeland, I. J.; Yuhanna, I. S.; Rader, D. R.; Lemos, J. A.; Shaul, P. W.; N. Engl. J. Med. 2014, 18, 2383.

3. Costa, L. G.; Cole, T. B.; Jarvik, G. P.; Furlong, C. E.; Аnпи. Rev. Med. 2003, 54,371 .

4. Hafiane, A.; Genest, J.; BBA Clin. 2015, 3, 175.

5. Marie, L.; Rached, F.; Le Goff, W.; Nègre-Salvayre, A.; Salvayre, R.; Calzada, C.; Lagarde, M.; Chapman, M. J.; Kontush, A.; Arterioscler. Thromb., Vasc. Biol. 2013, 33, 2715.

6. Asztalos, B. F.; Tani, M.; Schaefer, E. J.; Curr. Opin. Lipidol. 2011, 22, 176.
7. Saleheen, D.; Scott, R.; Javad, S.; Zhao, W.; Rodrigues, A.; Picataggi, A.; Lukmanova, D.; Mucksavage, M. L.; Luben, R.; Billheimer, J.; Kastelein, J. J.; Boekholdt, S. M.; Khaw, K. T.; Wareham, N.; Rader, D. J.; Lancet Diabetes Endocrinol. 2015, 3, 507.

8. Lima, E. S.; Maranhao, R. C.; Clin. Chem. 2004, 50, 1086

9. Friedewald, W. T.; Levy, R. I.; Fredrickson, D. S.; Clin. Chem. 1972, 18, 499.

10. Xavier, H. T.; Izar, M. C.; Faria-Neto J. R.; Assad, M. H.; Rocha, V. Z.; Sposito, A. C.; Fonseca, F. A.; dos Santos, J. E.; Santos R. D.; Bertolami, M. C.; Faludi A. A.; Martinez, T. L. R.; Diament, J.; Guimarães, A.; Forti, N. A.; Moriguchi, E.; Chagas, A. C. P.; Coelho, O. R.; Ramires, J. A. F.; Arq. Bras. Cardiol. 2013, 101, 1.

11. Mackness, M. I.; Harty, D.; Bhatnagar, D.; Winocour, P. H.; Arrol, S.; Ishola, M.; Durrington, P. N.; Atherosclerosis 1991, 86, 193.

12. Senti, M.; Tomas, M.; Fito, M.; Weinbrenner, T.; Covas, M. I.; Sala, J.; Masia, R.; Marrugat, J.; J. Clin. Endocrinol. Metab. 2003, 88, 5422.

13. Lewington, S.; Whitlock, G.; Clarke, R.; Sherliker, P.; Emberson, J.; Halsey, J.; Qizilbash, N.; Peto, R.; Collins, R.; Lancet 2007, 370, 1829.

14. Burstein, M.; Scholnick, H. R.; Morfin, R.; J. Lipid Res. 1970, 11, 583.

15. Srinivasan, S. R.; Radhakrishnamurthy, B.; Berenson, G. S.; Arch. Biochem. Biophys. 1975, 170, 334.

16. Eckerson, H. W.; Romson, J.; Wyte, C.; La Du B. N.; Am. J. Hum. Genet. 1983, 35, 214.

17. Schiavon, R.; De Fanti, E.; Giavarina, D.; Biasioli, S.; Cavalcanti, G.; Guidi, G.; Clin. Chim. Acta 1996, 29, 247.

18. Beltowisk, J; Wójcicka, G; Marcianiak, A.; Acta Biochim. Pol. 2002, 49, 927.

19. Davidson, W. S.; Heink, A.; Sexmith, H.; Melchior, J. T.; Gordon, S. M.; Kuklenyik, Z.; Woollett, L.; Barr, J. R.; Jones, J. I.; Toth, C. A.; Shah, A. S.; J. Lipid Res. 2016, 57, 674.

20. Dias, V. C.; Parsons, H. G.; Boyd, N. D.; Keane, P.; Clin. Chem. 1988, $34,2322$.

21. http://www.malvern.com/br/products/technology/light-scattering/ dynamic-light-scattering, acessada em agosto 2019.

22. Kim, Y. C.; Nishida, T.; J. Biol. Chem. 1979, 254, 9621. 